\title{
Diagnosis and staging of fibrosis in patients with chronic hepatitis C: comparison and critical overview of current strategies
}

This article was published in the following Dove Press journal:

Hepatic Medicine: Evidence and Research

\section{Leandro César Mendes Raquel SB Stucchi \\ Aline G Vigani}

Department of Infectious Diseases, State University of Campinas, Campinas, SP, Brazil
Correspondence: Leandro César Mendes Division of Infectious Diseases, Department of Internal Medicine, State University of Campinas, Cidade Universitária, I26 Tessalia Vieira de Camargo Street, Campinas City, SP |3084-97|, Brazil

Tel +551935217727

Fax +55 I9 353। 7727

Email lecmendes@yahoo.com.br

\begin{abstract}
In the past years, what has always been considered undisputed true in liver fibrosis staging has been challenged. Diagnostic performance of histological evaluation has proven to be significantly influenced by sample- and observer-related variabilities. Differentiation between lower levels of fibrosis remains difficult for many, if not all, test modalities, including liver biopsy but, perhaps, such a distinction is not indispensable in light of current therapeutic approaches. Biomarkers and elastography offer, nonetheless, high predictive values for advanced fibrosis and cirrhosis and correlate well with liver-related outcomes. Necroinflammation, steatosis, and hemodynamic changes may significantly interfere with elastography-based techniques, and longitudinal follow-up strategies must be tailored in light of these findings. Knowledge of different test modalities and diagnostic performance indicators can allow for better clinical decision-making and resource allocation.
\end{abstract}

Keywords: chronic hepatitis C, fibrosis, staging, elastography, biomarkers

\section{Introduction}

Chronic hepatitis $\mathrm{C}$ affects $\sim 70$ million people worldwide, representing one of the leading causes of liver-related death, hepatocellular carcinoma, and liver transplantation. ${ }^{1}$ The pathophysiological mechanism leading to such outcomes is sustained inflammation followed by progressive fibrosis and, ultimately, the vascular and architectural alterations of cirrhosis. Prompt diagnosis and management of advanced stages of fibrosis can prevent complications and death; however, optimal risk stratification is essential to avoid unnecessary and potentially wasteful resource allocation.

Liver fibrosis and inflammation in chronic hepatitis $\mathrm{C}$ are dynamic processes. Chronic hepatocellular damage, whether from direct cytophathic effect or through immunologic response, leads to hepatic stellate cell activation and alterations in extracellular matrix synthesis and degradation, including deposition of fibrillar collagen. In the normal liver, hepatic stellate cells are located in the space of Disse and store vitamin A. As chronic inflammation ensues, epigenetic phenomena partially initiated by platelet-derived growth factor secreted by Kupffer cells (and also by activation from other recruited immunological effectors such as $\mathrm{T}$ lymphocytes) lead to transdifferentiation into myofibroblast-like structures with inflammatory signaling, contractile, and fibrogenic properties migrating to specific sites of injury and repair, upregulating deposition and inhibiting degradation of extracellular matrix components. ${ }^{2,3}$ 
Histologically, fibrosis in chronic viral hepatitis starts within the portal tracts and spreads outward. As inflammation and persistent cell damage progress, the limiting plate is eroded and both hepatocyte death and fibrous scaring proceed permeating the adjoining parenchyma, usually through acinar region 1 and, in time, establishing fibrous septa between adjoining portal tracts or, less frequently, between a portal tract and a central vein. With more expansion of fibrous septa, hepatocytes become encompassed by septa and, eventually, begin to exhibit the disorganized regenerative nodular aspect of cirrhosis. In fact, instead of representing a static and inactive "scar", fibrous septa are also continuously changing. Early in the course of histological cirrhosis, septa are strongly infiltrated by mononuclear inflammatory cells and exhibit relatively thin fibrous content compared with larger regenerative hepatocyte nodules. Later, however, they become much thicker and less cellular, with neovascularization and micro porto-portal and porto-central shunting, as well as pronouncedly atrophic regenerative nodules. ${ }^{4}$

Faced with such diverse and dynamic processes, accurate staging presents a considerable challenge. Traditionally regarded as the gold standard, histological analysis through liver biopsy is not innocuous. More than that, it is subject to both observer- and sample-related biases and considerable variability. In the past two decades, different noninvasive approaches have been proposed and evaluated for liver fibrosis staging with sometimes conflicting reports on diagnostic accuracy and reproducibility. Several technical and patient characteristics affect the performance of different tests in different ways, as well as special patient populations with specific considerations.

Further, transitioning from an era when interferon was a therapeutic cornerstone, with discouraging chances of success and mounting adverse effects, to a period of directly acting antivirals offering both very high chances of sustained virologic response (SVR) and excellent safety profile, liver fibrosis staging as a triage for treatment indication may no longer be as pivotal as once believed..$^{5-7}$ Rather, recognizing advanced fibrosis and cirrhosis for comprehensive preventative and management strategies as well as tailoring treatment regimens may be much more important. Finally, such optimistic therapeutic scenario raises the issue of potential fibrosis regression across different levels and patient populations, as well as prognostic implications of fibrosis dynamics in patients after experiencing SVR.

Such topics will be reviewed in the following sections in light of current evidence and from a critical standpoint with emphasis on unresolved issues and their impact in $\mathrm{CHC}$ management.

\section{Fibrosis staging: What to search for and why?}

Pivotal studies have demonstrated long ago that fibrosis extending beyond portal spaces with few septa (corresponding to METAVIR level F2 and, more recently, referred to as significant fibrosis) is associated with some increase in risk of progression to cirrhosis. ${ }^{8-11}$ Conversely, it was assumed that patients in whom fibrosis was either absent or confined to porta spaces were, therefore, in relatively low risk of disease progression.

Interferon-based therapies resulted in adverse events in $>60 \%$ of patients, including flu-like symptoms, hematological, endocrine, and psychiatric manifestations, in some cases with considerable morbidity and, therefore, had several contraindications. Chances of SVR were, overall, $<50 \%$ after up to 72 weeks of therapy. ${ }^{12}$ In face of these safety and effectiveness issues, fibrosis staging guided treatment indication on the basis of unfavorable risk-to-benefit ratio. For those with METAVIR $\mathrm{F}<2$, low risk of progression deemed interferon-based therapy unjustifiable and they were advised to wait for future safer treatments.

In that setting, precise discrimination between adjacent levels of fibrosis (namely, F0, F1, and F2) was considered to be paramount for prompt treatment indication. This was largely based on the notion that fibrosis extending beyond portal spaces indicated an "active state" of chronic hepatitis $\mathrm{C}$ rather than a more benign inactive stage comprising METAVIR levels F0 or F1. Such a notion, however, was highly inferential and based on studies with small sample sizes, short follow-up intervals, and other methodological issues. More recently, however, larger cohorts have provided strong association between chronic hepatitis $\mathrm{C}$, irrespective of fibrosis stage, and increased risk of cardio and cerebrovascular events, among other non-liver-related morbidities as well as overall mortality compared with the general population. ${ }^{13-15}$

Directly acting antivirals have changed the landscape of CHC therapy, with SVR rates well over $90 \%$ and remarkably favorable safety profiles. ${ }^{16}$ Now, attributing risk-to-benefit ratios to most patient populations makes treatment contraindications few and scattered. Fibrosis staging to diagnose significant fibrosis (METAVIR $F \geq 2$ ), and sometimes, advanced fibrosis (METAVIR F $\geq 3$ ), continues to be used, however, to stratify risk mainly for population-level cost considerations in resource-limited settings.

If the diagnosis of significant fibrosis may have become somewhat superfluous in the current DAA-based therapy background, advanced fibrosis and cirrhosis continue to play a crucial role in $\mathrm{CHC}$ management. In terms of therapy 
tailoring, patients with cirrhosis have higher chances of achieving SVR with longer treatment durations and/or with the addition of ribavirin. ${ }^{17}$ Furthermore, it remains imperative to initiate screening strategies for hepatocellular carcinoma and clinically significant manifestations of portal hypertension in the cirrhotic population. In terms of treatment prioritization, longitudinal studies have shown that patients with advanced fibrosis and, especially, patients who have increasing results in annual follow-up are at increased risk of disease progression and death and, therefore, would benefit from prompt treatment initiation. ${ }^{18}$

As previously mentioned, cirrhosis is also dynamic in nature, and noninvasive testing, mainly elastography, has been shown to correlate well with risk of complications, including portal hypertension, variceal bleeding, hepatocellular carcinoma, and liver-related mortality. In this sense, recognizing different levels of fibrosis in the cirrhotic patient may be more useful both in clinical practice and in scientific research than differentiating between patients with absent or mild fibrosis and those with significant fibrosis which, in itself, has proven challenging for all fibrosis staging modalities, including histological analysis.

In the future, as DAA-based therapies become more accessible, diagnostic striving for accurate staging of patients with significant fibrosis tends to shift toward a more refined and detailed approach of the advanced fibrosis and cirrhosis population, with stronger risk stratification based on longitudinal variation of quantitative markers (such as liver stiffness), not limited to pretreatment evaluation, but going well beyond into the post-SVR period and lifelong patient management strategies.

\section{Liver biopsy: looking closer at the gold standard}

Histological assessment of liver biopsy fragments has long been considered the gold standard for fibrosis and necroinflammation in chronic hepatitis $\mathrm{C}$. The invasive nature of the procedure, however, can be an inconvenient and, sometimes, prohibitive, with up to $6 \%$ rate of overall complications $(75 \%$ of these patients reported persistent moderate to severe pain and $33 \%$ of cases presented with excessive bleeding requiring prolonged hospital observation and/or surgical intervention) and $0.03 \%$ risk of death. Coagulopathy, represented by low platelets or prolonged coagulation times, is an independent risk factor for adverse events. ${ }^{19}$

Furthermore, variability is a known and important issue for histological assessment, potentially compromising diagnostic performance. Percutaneous biopsy fragments represent about $1 / 50,000$ of the liver, being considered acceptable with $>15 \mathrm{~mm}$ in length and optimal with $>25 \mathrm{~mm}$ in length. Sampling variability between fragments taken from the left and right occurred in $33 \%$ of cases $(9.7 \%$ of which were classified as F0-F2 in one lobe and F3-F4 in the other). ${ }^{20,21}$

Observer-related issues are also common. The same liver biopsy fragment shown in different times to the same pathologist received different fibrosis staging in $25 \%$ of cases, and interobserver disagreement has been reported to be as high as $49 \%$. In $83 \%$ of cases where two blinded pathologists disagreed, fibrosis was understaged, one-third of which represented patients with advanced fibrosis (F3) who were erroneously classified as having mild or absent fibrosis (F1 or F0). ${ }^{22,23}$ When histological assessment was compared to digital morphometric collagen quantification in liver biopsy samples of different lengths, estimated area under receiver operating characteristic (AUROCs) for pathologist-based examination for adjacent METAVIR stages (F1 vs F0, F2 vs $\mathrm{F} 1$, and F3 vs F2) were, respectively, 0.56, 0.55, and 0.64 (for biopsy fragments of at least $15 \mathrm{~mm}$ ) and 0.56, 0.55, and 0.67 (for optimal fragments of at least $25 \mathrm{~mm}$ in length). ${ }^{24}$ Overall, diagnostic accuracy of histological evaluation was poorer in differentiating intermediate fibrosis stages and no significant improvement was observed among different levels of pathologist experience.

In terms of using histological analysis as a gold standard for diagnostic performance evaluation of comparator tests, an interesting mathematical model was proposed where AUROCs for any test compared to liver biopsy would be significantly influenced by the gold standard limitations and, for any scenario in which the sensitivity and specificity for histological analysis are $<90 \%$, it would be mathematically impossible for any comparator to be correctly evaluated, and this shows its true diagnostic potential. ${ }^{25}$ Notwithstanding, conventional histological assessment of liver biopsy is a time-honored method for evaluating liver disease, has been extensively validated, and is capable of providing key information regarding inflammation, possible comorbid pathological processes, fibrosis distribution, and deposits (such as iron and biliary pigments) as well as assessing important architectural information. In this sense, although limited in terms of variability and its invasive nature, liver biopsy remains an invaluable diagnostic tool in selected cases, as opposed to the previously almost universal method of choice as the initial test for fibrosis staging.

Digital morphometric analysis of liver biopsy fragments may provide an objective alternative, allowing for a broader and more detailed quantification of liver fibrosis correlating 
well with liver-related outcomes, including the recently proposed subclassifications of cirrhosis. Image acquisition is relatively simple with common microscopy equipment, and automated digital systems are available for image processing and collagen proportionate area quantification (Figure 1). ${ }^{26-29}$

\section{Noninvasive tests - biomarkers: from routine to research}

Different biomarkers have been associated with liver fibrosis. Some of them are "direct" as they are directly involved in extracellular matrix accumulation, whereas others are referred to as "indirect" for representing epiphenomena associated with the fibrogenic process as well as non-liver-specific inflammation.

Almost universally present in routine biochemical evaluation of liver diseases, aspartate aminotransferase (AST) and alanine aminotransferase (ALT) intracellular enzymes are released into serum as hepatocellular damage occurs. They are, nonetheless, present in other tissues and, therefore, isolated serum levels do not correlate perfectly with the extent of tissue injury. AST/ALT ratio, however, has been associated with advancing fibrosis soon after each individual marker was described in the 1950s. In chronic viral hepatitis, ALT levels usually rise disproportionately to AST, leading to AST/ALT ratio $<1.0$, and as fibrosis progresses, AST elevation becomes more pronounced and AST/ALT ratio becomes $>1.0$ and is directly proportional to advanced liver fibrosis and cirrhosis. In most studies, it is performed better in ruling out cirrhosis than for a positive diagnosis. Performance in lower levels of fibrosis has not been established. ${ }^{30-35}$

Also somewhat ubiquitous in routine laboratory testing, total platelet counts have been inversely correlated with advancing liver fibrosis. Several pathological mechanisms lead to lower platelet counts in patients with progressive liver fibrosis, including immune-mediated peripheral platelet destruction, inhibition of marrow production from diminished hepatic synthesis of growth factors, and increased spleen sequestering activity. ${ }^{36}$ At a threshold of 140 , platelet count had specificity $>90 \%$ significant fibrosis and $>85 \%$ for cirrhosis. ${ }^{37}$

Thus, AST to platelet ratio index (APRI) was proposed as an accessible biomarker score for predicting liver fibrosis comprising routinely obtained components. It has been extensively evaluated for diagnostic performance, consistently

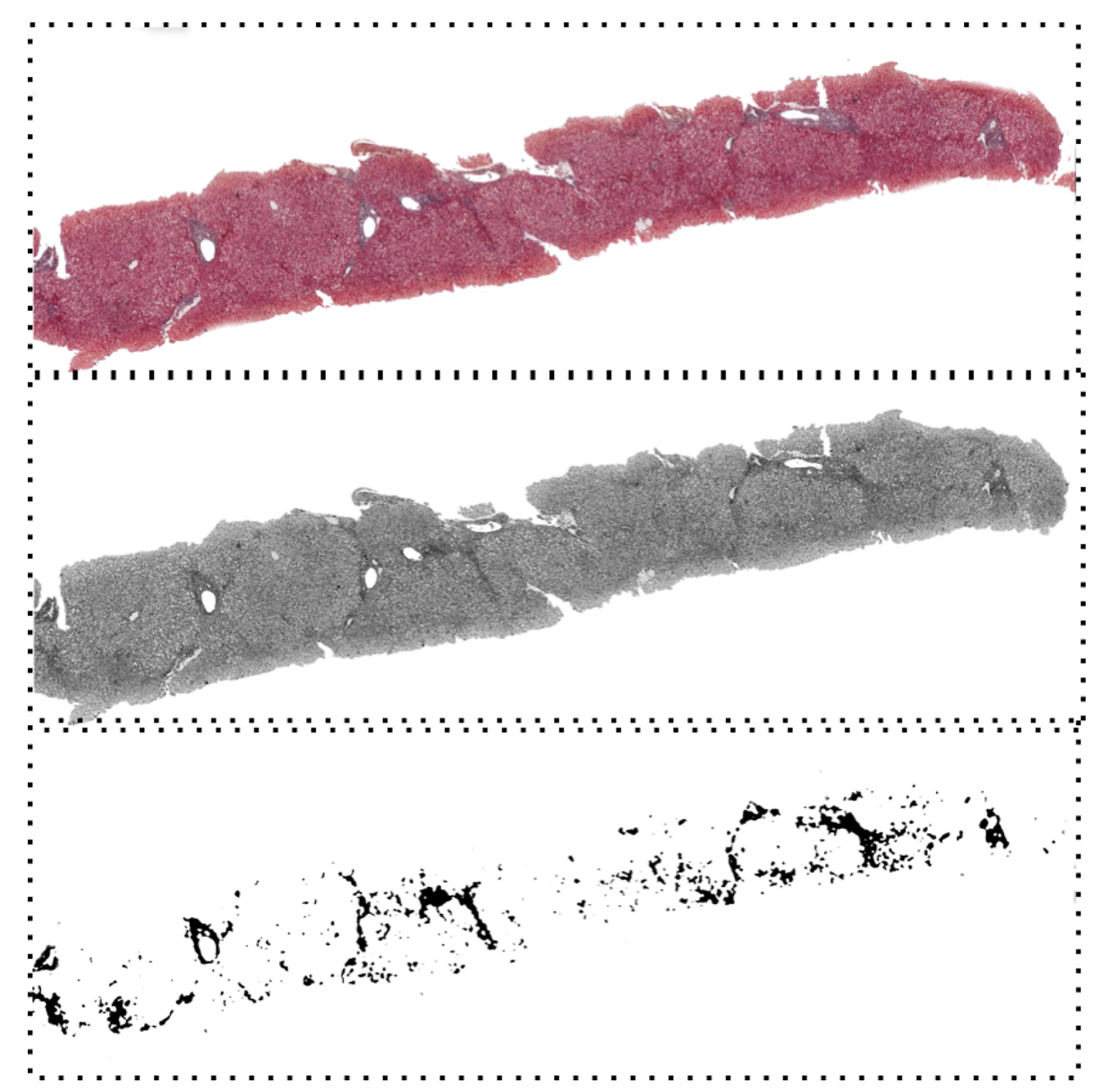

Figure I Digital morphometric analysis performed in Masson's trichrome-stained liver biopsy with collagen proportionate area quantified by image histogram analysis. 
exhibiting stronger positive diagnostic likelihood in advanced fibrosis and, especially, in cirrhosis. With optimal dual cutoff sensitivity for significant fibrosis if $<75 \% .{ }^{38-40}$ Other scores using similar components have been proposed, such as Fib4 (using ALT and age as well as AST and platelets, and having been initially validated also with a dual cutoff in the HIV/ hepatitis $\mathrm{C}$ virus [HCV]-coinfected population) ${ }^{41}$ and Forn's index (which includes total cholesterol as one of its components, along with gamma-glutamyltransferase, age, platelets, and prothrombin time and demonstrated a 0.81 AUROC for significant fibrosis), among others (Table 1). ${ }^{42}$

Other indirect biomarkers for liver fibrosis have been evaluated in logistic regressions. Among them, alpha-2- macroglobulin, haptoglobin, hyaluronic acid, apolipoproteins (and, also, total cholesterol), gamma glutamyl transferase, and total bilirubin were most strongly associated with different fibrosis stages and, therefore, were compiled in proprietary scores such as Fibrotest ${ }^{\circledR}$, Fibrometer $^{\circledR}$, and Hepascore ${ }^{\circledR}$. Alpha-2-macroglobulin is the best isolated predictor of liver fibrosis, and hyaluronic acid can be considered, in itself, a direct marker of fibrosis, being an integral part of extracellular matrix composition and accumulation. In general, individual tests also consistently performed best in advanced fibrosis/cirrhosis than in significant fibrosis. However, diagnostic accuracy in significant fibrosis discrimination has been greater than that observed for APRI or

Table I Tests capable of satisfactorily excluding/diagnosing significant fibrosis (negative and positive likelihood ratios $<0.25$ and $>5.0$, respectively)

\begin{tabular}{|c|c|c|c|c|}
\hline \multicolumn{5}{|c|}{ Excluding significant fibrosis } \\
\hline Biomarkers & Cut-off & Sensitivity & Specificity & Negative likelihood \\
\hline APRI ${ }^{39}$ & $\leq 0.36$ & $95 \%$ & $27 \%$ & 0.18 \\
\hline Forn's Index ${ }^{42}$ & $<4.2$ & $94 \%$ & $51 \%$ & 0.12 \\
\hline Fibroindex ${ }^{43}$ & $<1.25$ & $94 \%$ & $40 \%$ & 0.14 \\
\hline Fibrotest ${ }^{44}$ & $<0.22$ & $89 \%$ & $53 \%$ & 0.21 \\
\hline Fibrometer ${ }^{45}$ & $<0.44$ & $81 \%$ & $74 \%$ & 0.25 \\
\hline $\mathrm{ELF}^{46}$ & $<9.39$ & $90 \%$ & $55 \%$ & 0.19 \\
\hline \multicolumn{5}{|l|}{ Elastography } \\
\hline Fibroscan ${ }^{47}$ & $<5.2 \mathrm{kPa}$ & $97 \%$ & $35 \%$ & 0.09 \\
\hline 2D-SWE ${ }^{48}$ & $<7.1 \mathrm{kPa}$ & $90 \%$ & $89 \%$ & 0.11 \\
\hline PSWE (ARFI) ${ }^{49}$ & $<1.21 \mathrm{~m} / \mathrm{s}$ & $86 \%$ & $70 \%$ & 0.17 \\
\hline \multicolumn{5}{|l|}{ Combination } \\
\hline APRI+Fibrotest ${ }^{44}$ & $<0.5$ and $<0.22$ & $88 \%$ & $96 \%$ & 0.12 \\
\hline Fibrotest+Fibroscan ${ }^{50}$ & $<0.48$ and $<7.1 \mathrm{kPa}$ & $88 \%$ & $89 \%$ & 0.14 \\
\hline APRI+MP3 ${ }^{44}$ & $<1.0$ and $<0.30$ & $88 \%$ & 86 & 0.20 \\
\hline \multicolumn{5}{|c|}{ Diagnosing significant fibrosis } \\
\hline Biomarkers & Cut-off & Sensitivity & Specificity & Positive likelihood \\
\hline$\overline{\mathrm{APRI}} \mathrm{I}^{43}$ & $>0.85$ & $37 \%$ & $95 \%$ & 7.4 \\
\hline $\mathrm{FP}^{51}$ & $>0.8$ & $43 \%$ & $94 \%$ & 7.2 \\
\hline Fibrotest ${ }^{52}$ & $>0.48$ & $75 \%$ & $85 \%$ & 5.0 \\
\hline Fibroindex ${ }^{43}$ & $>2.25$ & $30 \%$ & $97 \%$ & 10.0 \\
\hline Forn's Index ${ }^{42}$ & $>6.9$ & $36 \%$ & $94 \%$ & 6.5 \\
\hline Hepascore $^{53}$ & $>0.5$ & $63 \%$ & $89 \%$ & 5.7 \\
\hline$M P 3^{54}$ & $>0.40$ & $35 \%$ & $96 \%$ & 8.75 \\
\hline \multicolumn{5}{|l|}{ Elastography } \\
\hline Fibroscan ${ }^{55}$ & $>7.1 \mathrm{kPa}$ & $67 \%$ & $89 \%$ & 6.1 \\
\hline 2D-SWE ${ }^{48}$ & $>7.1 \mathrm{kPa}$ & $90 \%$ & $89 \%$ & 7.2 \\
\hline PSWE $(A R F I)^{56}$ & $>1.34 \mathrm{~m} / \mathrm{s}$ & $68 \%$ & $93 \%$ & 9.7 \\
\hline \multicolumn{5}{|l|}{ Combination } \\
\hline Fibrotest+Fibroscan ${ }^{50}$ & $>0.48$ and $>7.1 \mathrm{kPa}$ & $85 \%$ & $97 \%$ & 7.8 \\
\hline APRI+Fibroscan ${ }^{57}$ & $>1.5$ and $>7.1 \mathrm{kPa}$ & $67 \%$ & $89 \%$ & 6.3 \\
\hline
\end{tabular}

Note: Information was based on individual studies compiled; no head-to-head comparisons were made. Tests and components: APRI: AST, platelets; Forn's index: Platelets, age, GGT, Total Cholesterol; Fibroindex: Platelets, AST, gamma globulin; Fibrotest ${ }^{\circledR}$ : Age, Total Bilirubin, GGT, Alfa2-macroglobulin, Haptoglobin, Apolipoprotein AI; Fibrometer ${ }^{\circledR}$ : Gender, Prothrombin time, GGT, Urea, Alfa2-macroglobulin, hyaluronate; ELF (Enhanced Liver Fibrosis score ${ }^{\circledR}$ ): PIIMP, TIMP-I and Hyaluronate; Hepascore ${ }^{\circledR}$ : Gender, GGT, Total Bilirubin, Hyaluronate, Alfa2-macroglobulin; MP3: PIIIMP and MMP-I; FPI: Age, AST, Total Cholesterol, insulin resistance, alcohol intake.

Abbreviations: 2D-SWE, two-dimensional shear-wave elastography; APRI, AST to platelet ratio index; AST, aspartate aminotransferase; ELF, enhanced liver fibrosis; FPI, fibrosis probability index; GGT, gamma glutamyl transferase; MMP-I, metalloproteinase I; PIIMP, N-terminal propeptide from type II collagen; pSWE (ARFI), point shear-wave elastography (acoustic radiation force impulse); TIMP-I, tissue inhibitor of metalloproteinase I. 
Fib-4. Head-to-head comparisons between these proprietary, more complex indexes have issued mixed results not allowing for any universal recommendation for preferential use between them.

Combinations of these scores have been attempted; Fibrotest and APRI were used in parallel or sequentially in three algorithms: SAFE biopsy, Leroy Algorithm, and Fibropaca. Overall, liver biopsy was indicated for discordant results and/or for exclusion of significant fibrosis when using traditional cutoff values for Fibrotest (0.48). Diagnostic performance was comparable, with AUROCs $>0.85$ for all outcomes, and saved liver biopsies from $29 \%$ to $79 \%$ of cases, depending on the specific algorithm. ${ }^{58,59}$

Substances directly associated with the fibrogenic process have been used alone or in combination to assess liver fibrosis. Most of them, however, remain experimental in nature and still lack widespread accessibility in clinical practice. Procollagen by-products such as N-terminal propeptide from types I and III collagen (PIMP and PIIINP) have been largely studied as markers of both inflammation and fibrosis and appear to correlate well with advanced fibrosis/cirrhosis (with 0.72 and 0.76 AUROCs, respectively). ${ }^{60-62}$ YKL-40, a glycoprotein associated with extracellular matrix remodeling expressed in hepatic stellate cells, has been found to correlate well with liver fibrosis with promising diagnostic performance in cirrhosis (AUROC 0.80). ${ }^{63,64}$ Finally, serum concentrations of metalloproteinases and their inhibitors (matrix metalloproteinases and tissue inhibitor of metalloproteinases [TIMPs]), involved in extracellular matrix degradation, have also been correlated with advanced fibrosis and cirrhosis, but with less reliable prediction capabilities in significant fibrosis. The most validated indexes combining direct markers are enhanced liver fibrosis (ELF), combining hyaluronic acid, TIMP, and N-terminal propeptide from type II collagen (PIINP) and MP3, combining PIIINP and metalloproteinase- 1 with AUROCs up to 0.81 and 0.88 for significant fibrosis and cirrhosis, respectively. ${ }^{54,65,66}$

Overall, diagnostic performances have been consistently better for cirrhosis prediction and exclusion across different markers and combinations, perhaps rendering several tests interchangeable. Significant fibrosis prediction, however, remains difficult. Among biomarkers, APRI with reduced cutoff, Forn's index, Fibrotest, Fibroindex, and ELF allow for significant fibrosis exclusion with negative likelihood $<0.25$.

\section{Noninvasive tests - elastography: Where do we stand on stiffness?}

Elastography refers to imaging techniques that quantify mechanical responses of tissues. Liver fibrosis alters the elastic properties of hepatic tissues, and various technologies have been developed in the past two decades to grade liver fibrosis as it correlates with tissue elastic response to deformation caused by an applied force (shear mechanical stress). Different modalities use different sources and nature of displacement wave and measure physical properties through different acquisition techniques. Currently, all elastographic techniques used to evaluate liver fibrosis measure shear-wave propagation speed and derive liver stiffness through Young's modulus.

Transient elastography is the most validated modality and the first publication for clinical use dates back to 2003. A piston produces a mechanical impulse on the skin surface generating shear waves that propagate through the liver. Shear-wave velocity is determined by a 3-MHz unidimensional ultrasound in a cylindrical area of interest with $10 \mathrm{~mm}$ in diameter and $40 \mathrm{~mm}$ in length located at varying distances from the origin point according to different probes (designed for pediatric, adult, and obese patients). Spatiotemporal propagation of the shear wave through the liver parenquima is represented graphically in an elastogram (different from the two-dimensional mapping images associated with B-mode-based ultrasound technologies). ${ }^{67,68}$ Measurements where shear-wave propagation is nonlinear or untraceable are considered invalid and not interpreted. Generated mechanical waves disperse in nonviscous liquids and, therefore, TE is not applicable to patients with ascites. Internal validation criteria are largely based on variability, although a quality score based on graphical properties of the shear-wave propagation representation has been found to predict accuracy. ${ }^{57}$ Diagnostic performance has been ascertained in comparison mostly to histological assessment of liver biopsy and has been consistently strongest in advanced fibrosis and cirrhosis, with sensitivity and specificity of $83 \%$ and $89 \%{ }^{69-72}$ Distinguishing between absent/ mild versus significant fibrosis appears to be more difficult, with sensitivity usually $<80 \%$. Similar results were obtained in $\mathrm{HIV} / \mathrm{HCV}$-coinfected patients, as well as those who have received orthotropic liver transplants. Necroinflammation and steatosis significantly increase liver stiffness and, therefore, may overestimate liver fibrosis staging. ${ }^{68,73}$

Similar to transient elastography in terms of generating shear waves through mechanical impulses to the surface of the skin, magnetic resonance elastography offers a unique opportunity to study the liver in its entirety, allowing for measurements in contexts where ultrasound-based technologies may be impractical, such as for patients with advanced obesity and very high skin-to-capsule distances. Diagnostic performances seem to be similar to those of other modalities; 
however, larger studies are necessary for further validation. Special equipment is required in addition to standard magnetic resonance devices, potentially hampering widespread access, particularly in resource-limited settings..$^{74,75}$

Acoustic Radiation Force Imaging technology is also employed for liver fibrosis staging as a source of an axial tissue exciting force. Point shear-wave propagation velocity is measured by ultrasound in a region of interest up to $8 \mathrm{~cm}$ deep from the skin surface, placed by the operator using B-mode ultrasound imaging. As with transient elastography, point shear-wave elastography-acoustic radiation force impulse (pSWE-ARFI) performs best in advanced fibrosis and cirrhosis (AUROCs $>0.85$ ) and somewhat more poorly in significant fibrosis (AUROC 0.79). ${ }^{76-79}$ Shear-wave speed can be measured continuously with real-time measurements in two-dimensional space and a much wider region of interest (two-dimensional shear-wave elastography [2D-SWE]). Also coupled with B-mode ultrasound imaging, 2D-SWE can be used in patients with ascites and has performed strongly for both significant and advanced fibrosis (AUROCs 0.95 and 0.96 , respectively) and for cirrhosis (AUROC 0.97) as well as with higher diagnostic accuracy compared with TE; however, larger studies are necessary to confirm such findings. ${ }^{48,80}$

Combination algorithms involving elastography (mainly TE) and biomarkers have been evaluated. The Bordeaux algorithm, using TE and Fibrotest, showed $88.3 \%$ and $94.2 \%$ accuracy in detecting significant fibrosis and cirrhosis, respectively. Similarly, associating Fibrometer and TE has accurately detected significant fibrosis and cirrhosis in $87.7 \%$ and $92.7 \%$ of patients. ${ }^{50}$

Regardless of the specific modality, liver stiffness correlates well with histological assessment of liver fibrosis and has demonstrated robust prognostic implications. Transient elastography predicted risk of death as an isolated measurement $(>9.5 \mathrm{kPa})$ and for those with positive variations over time. More than that, increasing liver stiffness was positively and continuously associated with incremental risk of death. ${ }^{18}$ Furthermore, liver stiffness correlated with the risk of developing hepatic complications, namely, clinically significant portal hypertension, hepatocellular carcinoma, and hepatic decompensation. In a prospective study, liver stiffness $<21.1$ $\mathrm{kPa}$ had $100 \%$ sensitivity for portal hypertension-related outcomes in chronic hepatitis C patients. ${ }^{81-83}$

Continued evaluation of patients who achieved SVR is a matter of debate. Most studies with relatively short follow-up intervals (usually $<3$ to 5 years) show consistent reductions in liver stiffness, most likely due to reduction in necroinflammatory activity and hemodynamic changes relative to decreases in portal pressure ${ }^{84}$ However, as the post-SVR patient population continues to rise, as well as concern for unknown risks such as new development or recurrence of hepatocellular carcinoma, questions regarding prolonged follow-up and long-term risk assessment are paramount and remain unanswered.

\section{Conclusion}

Noninvasive techniques for liver fibrosis staging in chronic hepatitis $\mathrm{C}$ have become increasingly available over the last decades. Diagnosis of significant fibrosis and distinction from mild/absent fibrosis are difficult across all test modalities (Table 1), including liver biopsy. Histological analysis limitations, especially observer-related variability, may have hampered diagnostic performance of comparator tests and, therefore, more objective quantification of liver fibrous tissue could provide a more reliable gold standard. Questions remain in terms of patient and technical factors interfering with diagnostic performance of different tests and possible ways to overcome them. With elastography-based tests or biomarkers, used alone or in combination, advanced fibrosis and cirrhosis are more easily identifiable (Tables 2 and 3 ) and,

Table 2 Tests capable of satisfactorily excluding/diagnosing advanced fibrosis (negative and positive likelihood ratios $<0.25$ and $>5.0$, respectively)

\begin{tabular}{|c|c|c|c|c|}
\hline \multicolumn{5}{|c|}{ Excluding advanced fibrosis } \\
\hline Biomarkers & Cut-off & Sensitivity & Specificity & $\begin{array}{l}\text { Negative } \\
\text { likelihood }\end{array}$ \\
\hline $\mathrm{APRI}^{39}$ & $<1.5$ & $87 \%$ & $50 \%$ & 0.26 \\
\hline Hepascore $^{53}$ & $<0.5$ & $88 \%$ & $74 \%$ & 0.16 \\
\hline $\mathrm{MP}^{54}$ & $<0.40$ & $92 \%$ & $58 \%$ & 0.14 \\
\hline \multicolumn{5}{|l|}{ Elastography } \\
\hline Fibroscan ${ }^{55}$ & $<8.0$ & $89 \%$ & $89 \%$ & 0.12 \\
\hline 2D-SWE ${ }^{48}$ & $<8.7$ & $97 \%$ & $95 \%$ & 0.03 \\
\hline pSWE (ARFI) ${ }^{49}$ & $<1.54$ & $97 \%$ & $100 \%$ & 0.03 \\
\hline \multicolumn{5}{|c|}{ Diagnosing advanced fibrosis } \\
\hline Biomarkers & Cut-off & Sensitivity & Specificity & $\begin{array}{l}\text { Positive } \\
\text { likelihood }\end{array}$ \\
\hline $\mathrm{APRI}^{39}$ & $>2.0$ & $36 \%$ & $93 \%$ & 5.2 \\
\hline $\mathrm{MP}^{54}$ & $>0.4$ & $58 \%$ & $92 \%$ & 7.25 \\
\hline Pohl Index ${ }^{85}$ & Positive & $41 \%$ & $89 \%$ & 5.36 \\
\hline $\mathrm{CDS}^{86}$ & $>8.0$ & $46 \%$ & $91 \%$ & 5.11 \\
\hline \multicolumn{5}{|l|}{ Elastography } \\
\hline Fibroscan 55 & $>9.5$ & $73 \%$ & $91 \%$ & 8.1 \\
\hline 2D-SWE ${ }^{48}$ & $>8.7$ & $97 \%$ & $95 \%$ & 19.7 \\
\hline pSWE $(A R F I)^{56}$ & $>1.6 \mathrm{I}$ & $79 \%$ & $95 \%$ & 15.3 \\
\hline
\end{tabular}

Note: Information based on individual studies compiled; no head-to-head comparisons were made. Tests and components: APRI: AST, platelets; Hepascore ${ }^{\circledast}$ : Gender, GGT, Total Bilirubin, Hyaluronate, Alfa2-macroglobulin; MP3: PIIMP and MMP-I; Pohl Index: AST/ALT, Platelets; CDS: AST/ALT, Platelets.

Abbreviations: 2D-SWE, two-dimensional shear-wave elastography; ALT, alanine aminotransferase; APRI, AST to platelet ratio index; AST, aspartate aminotransferase; GGT, gamma glutamyl transferase; MMP-I, metalloproteinase I; PIIMP, N-terminal propeptide from type II collagen; pSWE (ARFI), point shear-wave elastography (acoustic radiation force impulse). 
Table 3 Tests capable of satisfactorily excluding/diagnosing cirrhosis (negative and positive likelihood ratios $<0.25$ and $>5.0$, respectively)

\begin{tabular}{|c|c|c|c|c|}
\hline \multicolumn{5}{|c|}{ Excluding cirrhosis } \\
\hline Biomarkers & Cut-off & Sensitivity & Specificity & $\begin{array}{l}\text { Negative } \\
\text { likelihood }\end{array}$ \\
\hline Platelets $^{86}$ & $>150.000$ & $78 \%$ & $87 \%$ & 0.19 \\
\hline AST/ALT 85 & $<1.0$ & $77 \%$ & $97 \%$ & 0.22 \\
\hline Age to Platelets ${ }^{86}$ & $<6.0$ & $72 \%$ & $81 \%$ & 0.25 \\
\hline $\mathrm{APRI}^{39}$ & $<1.0$ & $84 \%$ & $72 \%$ & 0.22 \\
\hline Lok's index ${ }^{87}$ & $<0.2$ & $92 \%$ & $50 \%$ & 0.16 \\
\hline $\mathrm{ELF}^{46}$ & $<9.8$ & $97 \%$ & $59 \%$ & 0.05 \\
\hline \multicolumn{5}{|l|}{ Imaging } \\
\hline Fibroscan ${ }^{55}$ & $<11.6$ & $92 \%$ & $97 \%$ & 0.09 \\
\hline 2D-SWE ${ }^{48}$ & $<10.4$ & $87 \%$ & $97 \%$ & 0.13 \\
\hline pSWE (ARFI) ${ }^{56}$ & $<2.00$ & $80 \%$ & $95 \%$ & 0.21 \\
\hline \multicolumn{5}{|c|}{ Diagnosing cirrhosis } \\
\hline Biomarkers & Cut-off & Sensitivity & Specificity & $\begin{array}{l}\text { Positive } \\
\text { likelihood }\end{array}$ \\
\hline Platelets $^{86}$ & $<140.000$ & $78 \%$ & $87 \%$ & 6.0 \\
\hline Age to Platelets ${ }^{86}$ & $>6.0$ & $70 \%$ & $88 \%$ & 5.8 \\
\hline $\mathrm{APRI}^{39}$ & $>2.0$ & $51 \%$ & $94 \%$ & 8.5 \\
\hline Lok's index ${ }^{87}$ & $>0.7$ & $11 \%$ & $98 \%$ & 5.5 \\
\hline Fibrotest ${ }^{88}$ & $>0.75$ & $50 \%$ & $93 \%$ & 5.11 \\
\hline ELF $^{46}$ & $>11.3$ & $83 \%$ & $97 \%$ & 28.0 \\
\hline \multicolumn{5}{|l|}{ Imaging } \\
\hline US surface & Positive & $54 \%$ & $95 \%$ & 11.6 \\
\hline \multicolumn{5}{|l|}{ nodularity ${ }^{89}$} \\
\hline Fibroscan 90 & $>14.6 \mathrm{kPa}$ & $86 \%$ & $96 \%$ & 21.0 \\
\hline 2D-SWE ${ }^{48}$ & $>10.4 \mathrm{kPa}$ & $88 \%$ & $97 \%$ & 28.0 \\
\hline pSWE (ARFI) ${ }^{56}$ & $>2.00$ & $80 \%$ & $95 \%$ & 17.6 \\
\hline
\end{tabular}

Note: Information based on individual studies compiled; no head-to-head comparisons were made. Tests and components: APRI: AST, platelets; Lok index: Platelets, AST/ALT, INR; ELF (Enhanced Liver Fibrosis score ${ }^{\circledR}$ ): PIIMP, TIMPI, and Hyaluronate; Fibrotest ${ }^{\circledR}$ : Age, Total Bilirubin, GGT, Alfa2-macroglobulin, Haptoglobin, Apolipoprotein AI.

Abbreviations: 2D-SWE, two-dimensional shear-wave elastography; ALT, alanine aminotransferase; APRI, AST to platelet ratio index; AST, aspartate aminotransferase; ELF, enhanced liver fibrosis; GGT, gamma glutamyl transferase; INR, international normalized ratio; PIIMP, N-terminal propeptide from type II collagen; pSWE (ARFI), point shear-wave elastography (acoustic radiation force impulse); TIMP-I, tissue inhibitor of metalloproteinase I; US, conventional ultrasound.

furthermore, they allow for risk stratification for individualized therapeutic and surveillance approaches.

\section{Disclosure}

The authors report no conflicts of interest in this work.

\section{References}

1. Polaris Observatory HCV Collaborators. Global prevalence and genotype distribution of hepatitis $\mathrm{C}$ virus infection in 2015: a modelling study. Lancet Gastroenterol Hepatol. 2017;2:161-176.

2. Bataller R, Brenner DA. Liver fibrosis. JClin Invest. 2005;115(2):209-218.

3. Tsukada S, Parsons CJ, Rippe RA. Mechanisms of liver fibrosis. Clin Chim Acta. 2006;364(1-2):33-60.
4. Garcia-Tsao G, Friedman S, Iredale J, Pinzani M. Now there are many (stages) where before there was one: in search of a pathophysiological classification of cirrhosis. Hepatology. 2010;51(4):1445-1449.

5. Carmona I, Cordero P, Ampuero J, Rojas A, Romero-Gómez M. Role of assessing liver fibrosis on management of chronic hepatitis $\mathrm{C}$ virus infection. Clin Microbiol Infect. 2016;22(10):839-845.

6. Tangkijvanich P, Chittmittraprap S, Poovorawan K, et al. A randomized clinical trial of peginterferon alpha-2b with or without entecavir in patients with $\mathrm{HBeAg}$-negative chronic hepatitis B: role of host and viral factors associated with treatment response. J Viral Hepat. 2016;23(6):427-438.

7. Bunchorntavakul C, Reddy KR. Management of hepatitis C before and after liver transplantation in the era of rapidly evolving therapeutic advances. J Clin Transl Hepatol. 2014;2(2):124-133.

8. Ikeda K, Saitoh S, Suzuki Y, et al. Disease progression and hepatocellular carcinogenesis in patients with chronic viral hepatitis: a prospective observation of 2215 patients. $J$ Hepatol. 1998;28(6):930-938.

9. Niederau C, Lange S, Heintges T, et al. Prognosis of chronic hepatitis C: results of a large, prospective cohort study. Hepatology. 1998;28(6): $1687-1695$.

10. Yano M, Kumada H, Kage M, et al. The long-term pathological evolution of chronic hepatitis C. Hepatology. 1996;23(6):1334-1340.

11. Khan M, Farrell G, Byth K, et al. Which patients with hepatitis C develop liver complications? Hepatology. 2000;31(2):513-520.

12. Fried MW. Sido effects of therapy of hepatitis $\mathrm{C}$ and their management. Hepatology. 2002;36(5 suppl 1):S237-S244.

13. Petta S, Maida M, Macaluso F, et al. Hepatitis $C$ virus infection is associated with increased cardiovascular mortality: a meta-analysis of observational studies. Gastroenterology. 2016;150(1):145-155.

14. Cepeda J, Thomas D, Astemborski J, Sulkowski M, Kirk G, Mehta $\mathrm{S}$. Increased mortality among persons with chronic hepatitis $\mathrm{C}$ with moderate or severe liver disease: a cohort study. Clin Infect Dis. 2017;65(2):235-243.

15. Hsu Y, Ho H, Huang Y, et al. Association between antiviral treatment and extrahepatic outcomes in patients with hepatitis $\mathrm{C}$ virus infection. Gut. 2014;64(3):495-503.

16. Banerjee D, Reddy K. Review article: safety and tolerability of directacting anti-viral agents in the new era of hepatitis C therapy. Aliment Pharmacol Ther. 2016;43(6):674-696.

17. Poordad F, Schiff E, Vierling J, et al. Daclatasvir with sofosbuvir and ribavirin for hepatitis $\mathrm{C}$ virus infection with advanced cirrhosis or postliver transplantation recurrence. Hepatology. 2016;63(5):1493-1505.

18. Vergniol J, Foucher J, Terrebonne E, et al. Noninvasive tests for fibrosis and liver stiffness predict 5-year outcomes of patients with chronic hepatitis C. Gastroenterology. 2011;140(7):1970-1979.

19. Chi H, Hansen B, Tang W, et al. Multiple biopsy passes and the risk of complications of percutaneous liver biopsy. Eur J Gastroenterol Hepatol. 2017;29(1):36-41.

20. Bedossa P, Dargere D, Paradis V. Sampling variability of liver fibrosis in chronic hepatitis C. Hepatology. 2003;38(6):1449-1457.

21. Regev A, Berho M, Jeffers L, et al. Sampling error and intraobserver variation in liver biopsy in patients with chronic $\mathrm{HCV}$ infection. Am J Gastroenterol. 2002;97(10):2614-2618.

22. Cholongitas E, Senzolo M, Standish R, et al. A systematic review of the quality of liver biopsy specimens. Am J Clin Pathol. 2006;125(5): 710-721.

23. Chindamo MC, Nunes-Pannain VL, Araújo-Neto JM, et al. Intermediate fibrosis staging in hepatitis $\mathrm{C}$ : a problem not overcome by optimal samples or pathologists' expertise. Ann Hepatol. 2015;14(5):652-657.

24. Poynard T, Lenaour G, Vaillant J, et al. Liver biopsy analysis has a low level of performance for diagnosis of intermediate stages of fibrosis. Clin Gastroenterol Hepatol. 2012;10(6):657-663.

25. Mehta S, Lau B, Afdhal N, Thomas D. Exceeding the limits of liver histology markers. J Hepatol. 2009;50(1):36-41.

26. Kirk G, Astemborski J, Mehta S, et al. Assessment of liver fibrosis by transient elastography in persons with hepatitis $\mathrm{C}$ virus infection or HIV-hepatitis C virus coinfection. Clin Infect Dis. 2009;48(7):963-972. 
27. Calès P, Chaigneau J, Hunault G, et al. Automated morphometry provides accurate and reproducible virtual staging of liver fibrosis in chronic hepatitis C. J Pathol Inform. 2015;6(1):1-20.

28. Calvaruso V, Di Marco V, Bavetta M, et al. Quantification of fibrosis by collagen proportionate area predicts hepatic decompensation in hepatitis C cirrhosis. Aliment Pharm Ther. 2015;41(5):477-486.

29. Calvaruso V, Burroughs A, Standish R, et al. Computer-assisted image analysis of liver collagen: relationship to Ishak scoring and hepatic venous pressure gradient. Hepatology. 2008;49(4):1236-1244.

30. Williams AL, Hoofnagle JH. Ratio of serum aspartate to alanine aminotransferase in chronic hepatitis: relationship to cirrhosis. Gastroenterology. 1988;95(3):734-739.

31. Sheth SG, Flamm SL, Gordon FD, Chopra S. AST/ALT ratio predicts cirrhosis in patients with chronic hepatitis $\mathrm{C}$ virus infection. $\mathrm{Am} \mathrm{J}$ Gastroenterol. 1998;93:44-48.

32. Reedy DW, Loo AT, Levine RA. AST/ALT ratio $\geq 1$ is not diagnostic of cirrhosis in patients with chronic hepatitis C. Dig Dis Sci. 1998;43(9):2156-2159.

33. Park GJH, Lin BPC, Ngu MC, Jones DB, Katelaris PH. Aspartate aminotransferase alanine aminotransferase ratio in chronic hepatitis $\mathrm{C}$ infection: is it a useful predictor of cirrhosis? J Gastroenterol Hepatol. 2000;15(4):386-390

34. Imperiale TF, Said AT, Cummings OW, Born LJ. Need for validation of clinical decision aids: use of the AST/ALT ratio in predicting cirrhosis in chronic hepatitis C. Am J Gastroenterol. 2000;95:2328-2332.

35. Giannini E, Risso D, Botta F, et al. Validity and clinical utility of the aspartate aminotransferase-alanine aminotransferase ratio in assessing disease severity and prognosis in patients with hepatitis $\mathrm{C}$ virus-related chronic liver disease. Arch Intern Med. 2003;163(2):218-224.

36. Peck-Radosavljevic M. Thrombocytopenia in chronic liver disease. Liver Int. 2017;37(6):778-793.

37. Ono E, Shiratori Y, Okudaira T, et al. Platelet count reflects stage of chronic hepatitis C. Hepatol Res. 1999;15:192-200.

38. Wai C, Greenson JK, Fontana RJ, et al. A simple noninvasive index can predict both significant fibrosis and cirrhosis in patients with chronic hepatitis C. Hepatology. 2003;38(2):518-526.

39. Lin Z, Xin Y, Dong Q, et al. Performance of the aspartate aminotransferase-to-platelet ratio index for the staging of hepatitis C-related fibrosis: an updated meta-analysis. Hepatology. 2011;53(3):726-736.

40. Shaheen A, Myers R. Diagnostic accuracy of the aspartate aminotransferase-to-platelet ratio index for the prediction of hepatitis C-related fibrosis: a systematic review. Hepatology. 2007;46(3):912-921.

41. Sterling, R, Lissen, E, Clumeck, N, et al; APRICOT Clinical Investigators. Development of a simple noninvasive index to predict significant fibrosis in patients with HIV/HCV coinfection. Hepatology. 2006;43(6):1317-1325.

42. Forns $X$. Identification of chronic hepatitis $C$ patients without hepatic fibrosis by a simple predictive model. Hepatology. 2002;36(4):986-992.

43. Koda M, Matunaga Y, Kawakami M, Kishimoto Y, Suou T, Murawaki Y. Fibroindex, a practical index for predicting significant fibrosis in patients with chronic hepatitis C. Hepatology. 2007;45(2):297-306.

44. Leroy V, Hilleret M, Sturm N, et al. Prospective comparison of six noninvasive scores for the diagnosis of liver fibrosis in chronic hepatitis $\mathrm{C}$. J Hepatol. 2007;46(5):775-782.

45. Calès P, Boursier J, Bertrais S, et al; Multicentric groups (SNIFF 14 \& 17, ANRS HC EP 23 Fibrostar). Optimization and robustness of blood tests for liver fibrosis and cirrhosis. Clin Biochem. 2010;43(16-17):1315-1322.

46. Parkes J, Guha I, Roderick P, et al. Enhanced Liver Fibrosis (ELF) test accurately identifies liver fibrosis in patients with chronic hepatitis $\mathrm{C}$. J Viral Hepat. 2010;18(1):23-31.

47. Zarski J, Sturm N, Guechot J, et al; ANRS HCEP 23 Fibrostar Group. Comparison of nine blood tests and transient elastography for liver fibrosis in chronic hepatitis C: the ANRS HCEP-23 study. $J$ Hepatol. 2012;56(1):55-62.

48. Ferraioli G, Tinelli C, Dal Bello B, Zicchetti M, Filice G, Filice C; Liver Fibrosis Study Group. Accuracy of real-time shear wave elastography for assessing liver fibrosis in chronic hepatitis C: a pilot study. Hepatology. 2012;56(6):2125-2133.
49. Fierbinteanu-Braticevici C. Acoustic radiation force imaging sonoelastography for noninvasive staging of liver fibrosis. World J Gastroenterol. 2009;15(44):5525-5532.

50. Boursier J, de Ledinghen V, Zarski J, et al; Multicentric groups from SNIFF 32, VINDIAG 7, and ANRS/HC/EP23 FIBROSTAR studies. Comparison of eight diagnostic algorithms for liver fibrosis in hepatitis $\mathrm{C}$ : new algorithms are more precise and entirely noninvasive. Hepatology. 2012;55(1):58-67.

51. Sud A, Hui J, Farrell G, et al. Improved prediction of fibrosis in chronic hepatitis $\mathrm{C}$ using measures of insulin resistance in a probability index. Hepatology. 2004;39(5):1239-1247.

52. Imbert-Bismut F, Ratziu V, Pieroni L, Charlotte F, Benhamou Y, Poynard T. Biochemical markers of liver fibrosis in patients with hepatitis $\mathrm{C}$ virus infection: a prospective study. Lancet. 2001;357(9262):1069-1075.

53. Adams L, Bulsara M, Rossi E, et al. Hepascore: an accurate validated predictor of liver fibrosis in chronic hepatitis $\mathrm{C}$ infection. Clin Chem. 2005;51(10):1867-1873.

54. Leroy V, Monier F, Bottari S, et al. Circulating matrix metalloproteinases 1, 2, 9 and their inhibitors TIMP-1 and TIMP-2 as serum markers of liver fibrosis in patients with chronic hepatitis $\mathrm{C}$ : comparison with PIIINP and hyaluronic acid. Am J Gastroenterol. 2004;99(2): 271-279.

55. Castéra L, Vergniol J, Foucher J, et al. Prospective comparison of transient elastography, Fibrotest, APRI, and liver biopsy for the assessment of fibrosis in chronic hepatitis C. Gastroenterology. 2005;128(2):343-350.

56. Lupsor M, Badea R, Stefanescu H, et al. Performance of a new elastographic method (ARFI technology) compared to unidimensional transient elastography in the noninvasive assessment of chronic hepatitis C. Preliminary results. J Gastrointestin Liver Dis. 2009;18(3):303-310.

57. Mendes LC, Ferreira PA, Miotto N, et al. Elastogram quality assessment score in vibration-controlled transient elastography: diagnostic performance compared to digital morphometric analysis of liver biopsy in chronic hepatitis C. J Viral Hepat. Epub 2017 Nov 1.

58. Sebastiani G, Halfon P, Castéra L, et al. Comparison of three algorithms of non-invasive markers of fibrosis in chronic hepatitis C. Aliment Pharmacol Ther. 2012;35(1):92-104.

59. Castéra L, Sebastiani G, Le Bail B, de Lédinghen V, Couzigou P, Alberti A. Prospective comparison of two algorithms combining non-invasive methods for staging liver fibrosis in chronic hepatitis C. $J$ Hepatol. 2010;52(2):191-198.

60. Nøjgaard C, Johansen JS, Christensen E, Skovgaard LT, Price PA, Becker U; EMALD Group. Serum levels of YKL-40 and PIIINP as prognostic markers in patients with alcoholic liver disease. $J$ Hepatol. 2003;39(2):179-186.

61. Rosenberg WM, Voelker M, Thiel R, et al; European Liver Fibrosis Group. Serum markers detect the presence of liver fibrosis: a cohort study. Gastroenterology. 2004;127(6):1704-1713.

62. Schytte S, Hansen M, Møller S, et al. Hepatic and renal extraction of circulating type I procollagen aminopropeptide in patients with normal liver function and in patients with alcoholic cirrhosis. Scand J Clin Lab Invest. 1999;59(8):627-633.

63. Tran A, Benzaken S, Saint-Paul MC, et al. Chondrex (YKL-40), a potential new serum fibrosis marker in patients with alcoholic liver disease. Eur J Gastroenterol Hepatol. 2000;12(9):989-993.

64. Saitou Y, Shiraki K, Yamanaka Y, et al. Noninvasive estimation of liver fibrosis and response to interferon therapy by a serum fibrogenesis marker, YKL-40, in patients with HCV associated liver disease. World J Gastroenterol. 2005;11(4):476-481.

65. Boeker KH, Haberkorn CI, Michels D, Flemming P, Manns MP, Lichtinghagen R. Diagnostic potential of circulating TIMP-1 and MMP-2 as markers of liver fibrosis in patients with chronic hepatitis C. Clin Chim Acta. 2002;316(1-2):71-81.

66. Walsh KM, Timms P, Campbell S, MacSween RN, Morris AJ. Plasma levels of matrix metalloproteinase-2 (MMP-2) and tissue inhibitors of metalloproteinases -1 and -2 (TIMP-1 and TIMP-2) as noninvasive markers of liver disease in chronic hepatitis C: comparison using ROC analysis. Dig Dis Sci. 1999;44(3):624-630. 
67. Sandrin L, Fourquet B, Hasquenoph J, et al. Transient elastography: a new noninvasive method for assessment of hepatic fibrosis. Ultrasound Med Biol. 2003;29(12):1705-1713.

68. Wong GL. Update of liver fibrosis and steatosis with transient elastography (Fibroscan). Gastroenterol Rep. 2013;1(1):19-26.

69. Seo Y, Kim M, Kim S, et al; Korean Transient Elastography Study Group. Accuracy of transient elastography in assessing liver fibrosis in chronic viral hepatitis: a multicentre, retrospective study. Liver Int. 2015;35(10):2246-2255.

70. Friedrich-Rust M, Ong M, Martens S, et al. Performance of transient elastography for the staging of liver fibrosis: a meta-analysis. Gastroenterology. 2008;134(4):960-974.

71. Chang PE, Goh GB, Ngu JH, Tan HK, Tan CK. Clinical applications, limitations and future role of transient elastography in the management of liver disease. World J Gastrointest Pharmacol Ther. 2016;7:91-106.

72. Tsochatzis EA, Gurusamy KS, Ntaoula S, Cholongitas E, Davidson BR, Burroughs AK. Elastography for the diagnosis of severity of fibrosis in chronic liver disease: a meta-analysis of diagnostic accuracy. $J$ Hepatol. 2011;54(4):650-659.

73. Macaluso F, Maida M, Cammà C, et al. Steatosis affects the performance of liver stiffness measurement for fibrosis assessment in patients with genotype 1 chronic hepatitis C. J Hepatol. 2014;61(3):523-529.

74. Huwart L, Sempoux C, Vicaut E, et al. Magnetic resonance elastography for the noninvasive staging of liver fibrosis. Gastroenterol. 2008;135:32-40.

75. Singh S, Venkatesh SK, Wang Z, et al. Diagnostic performance of magnetic resonance elastography in staging liver fibrosis: a systematic review and meta-analysis of individual participant data. Clin Gastroenterol Hepatol. 2015;13:440-451.

76. Friedrich-Rust M, Wunder K, Kriener S, et al. Liver fibrosis in viral hepatitis: noninvasive assessment with acoustic radiation force impulse imaging versus transient elastography. Radiology. 2009;252(2):595-604.

77. Sporea I, Bota S, Peck-Radosavljevic M, et al. Acoustic Radiation Force Impulse elastography for fibrosis evaluation in patients with chronic hepatitis C: an international multicenter study. Eur J Radiol. 2012;81(12):4112-4118.

78. Friedrich-Rust M, Lupsor M, de Knegt R, et al. Point shear wave elastography by acoustic radiation force impulse quantification in comparison to transient elastography for the noninvasive assessment of liver fibrosis in chronic hepatitis $\mathrm{C}$ : a prospective international multicenter study. Ultraschall in Med. 2015;36(3):239-247.
79. Friedrich-Rust M, Nierhoff J, Lupsor M, et al. Performance of Acoustic Radiation Force Impulse imaging for the staging of liver fibrosis: a pooled meta-analysis. J Viral Hepat. 2012;19(2):e212-e219.

80. Tada T, Kumada T, Toyoda H, et al. Utility of real-time shear wave elastography for assessing liver fibrosis in patients with chronic hepatitis $\mathrm{C}$ infection without cirrhosis: comparison of liver fibrosis indices. Hepatol Res. 2015;45(10):e122-e129.

81. Masuzaki R, Tateishi R, Yoshida H, et al. Prospective risk assessment for hepatocellular carcinoma development in patients with chronic hepatitis C by transient elastography. Hepatology. 2009;49(6):1954-1961.

82. Poynard T, Vergniol J, Ngo Y, et al; FibroFrance Study Group; Epic3 Study Group; Bordeaux HCV Study Group. Staging chronic hepatitis $\mathrm{C}$ in seven categories using fibrosis biomarker (FibroTest) and transient elastography (FibroScan(R)). J Hepatol. 2014;60(4):706-714.

83. Robic M, Procopet B, Métivier S, et al. Liver stiffness accurately predicts portal hypertension related complications in patients with chronic liver disease: a prospective study. J Hepatol. 2011;55(5):1017-1024.

84. Singh S, Facciorusso A, Loomba R, Falck-Ytter YT. Magnitude and kinetics of decrease in liver stiffness after antiviral therapy in patients with chronic hepatitis $\mathrm{C}$ : a systematic review and meta-analysis. Clin Gastroenterol Hepatol 2018;16(1):27.e4-38.e4.

85. Pohl A, Behling C, Oliver D, Kilani M, Monson P, Hassanein T. Serum aminotransferase levels and platelet counts as predictors of degree of fibrosis in chronic hepatitis C virus infection. Am J Gastroenterol. 2001;96(11):3142-1346.

86. Lackner C, Struber G, Liegl B, et al. Comparison and validation of simple noninvasive tests for prediction of fibrosis in chronic hepatitis C. Hepatology. 2005;41(6):1376-1382.

87. Lok A, Ghany M, Goodman Z, et al. Predicting cirrhosis in patients with hepatitis $\mathrm{C}$ based on standard laboratory tests: results of the HALT-C cohort. Hepatology. 2005;42(2):282-292.

88. Shaheen A, Wan A, Myers R. FibroTest and FibroScan for the prediction of hepatitis C-related fibrosis: a systematic review of diagnostic test accuracy. Am J Gastroenterol. 2007;102(11):2589-2600.

89. Colli A, Fraquelli M, Andreoletti M, Marino B, Zuccoli E, Conte D. Severe liver fibrosis or cirrhosis: accuracy of US for detection-analysis of 300 cases. Radiology. 2003;227(1):89-94.

90. Ziol M, Handra-Luca A, Kettaneh A, et al. Noninvasive assessment of liver fibrosis by measurement of stiffness in patients with chronic hepatitis C. Hepatology. 2005;41(1):48-54.
Hepatic Medicine: Evidence and Research

\section{Publish your work in this journal}

Hepatic Medicine: Evidence and Research is an international, peerreviewed, open access journal covering all aspects of adult and pediatric hepatology in the clinic and laboratory including the following topics: Pathology, pathophysiology of hepatic disease; Investigation and treatment of hepatic disease; Pharmacology of drugs used for the treatment
Dovepress

of hepatic disease. Issues of patient safety and quality of care will also be considered. The manuscript management system is completely online and includes a very quick and fair peer-review system, which is all easy to use. Visit http://www.dovepress.com/testimonials.php to read real quotes from published authors. 